\title{
Diaphragm electromyogram measured with unilateral magnetic stimulation
}

\author{
Y.M. Luo*, L.C. Johnson*, M.I. Polkey*, M.L. Harris*, R.A. Lyall*, M. Green ${ }^{+}$, J. Moxham*
}

Diaphragm electromyogram measured with unilateral magnetic stimulation. Y.M. Luo, L.C. Johnson, M.I. Polkey, M.L. Harris, R.A. Lyall, M. Green, J. Moxham. (C)ERS Journals Ltd 1999.

ABSTRACT: The purpose of this study was to establish the phrenic nerve conduction time (PNCT) for magnetic stimulation and further assess the relatively new technique of anterior unilateral magnetic stimulation (UMS) of the phrenic nerves in evaluating the diaphragm electromyogram (EMG).

An oesophageal electrode was used to record the diaphragm compound muscle action potential (CMAP) elicited by supramaximal percutaneous electrical phrenic nerve stimulation (ES) and UMS from eight normal subjects. The oesophageal electrode used for recording the CMAP was positioned at the level of the hiatus and 3 cm below. The diaphragm CMAP was also recorded from chest wall surface electrodes in five subjects.

All of the phrenic nerves could be maximally stimulated with UMS. A clear plateau of the amplitude of the CMAP was achieved for the right and left phrenic nerves. The mean amplitudes of the CMAP recorded from the oesophageal electrode were, for the right side, $0.74 \pm 0.29 \mathrm{mV}$ (mean $\pm \mathrm{SD})$ for $\mathrm{ES}$ and $0.76 \pm 0.30 \mathrm{mV}$ for UMS with maximal power output, and for the left side $0.88 \pm 0.33 \mathrm{mV}$ for $\mathrm{ES}$ and $0.80 \pm 0.24 \mathrm{mV}$ for UMS. PNCT measured by the oesophageal electrode with ES and UMS with maximal output were, for the right side, $7.0 \pm 0.8 \mathrm{~ms}$ and $6.9 \pm 0.8 \mathrm{~ms}$, respectively, and for the left side $7.8 \pm 1.2 \mathrm{~ms}$ and $7.7 \pm 1.3 \mathrm{~ms}$, respectively. However, the CMAP recorded from chest wall surface electrodes with UMS was unsuitable for the measurement of PNCT.

The results suggest that unilateral magnetic stimulation of the phrenic nerves combined with an oesophageal electrode can be used to assess diaphragmatic electrical activity and measure the phrenic nerve conduction time.

Eur Respir J 1999; 13: 385-390.
*King's College Hospital and ${ }^{+}$Royal Brompton Hospital, London, UK.

Correspondence: Y.M. Luo

Respiratory Muscle Laboratory

King's College School of Medicine and Dentistry

Bessemer Road

London SE5 9PJ

UK

Fax: 441713463589

Keywords: Magnetic stimulation oesophageal electrode

phrenic nerve conduction time

Received: June 181998

Accepted after revision September 241998
Measuring the phrenic nerve conduction time (PNCT) and the diaphragm electromyogram (EMG) in response to phrenic nerve stimulation provides useful information for the assessment of diaphragm function and in the diagnosis of neuromuscular disease. PNCT measured with conventional electrical stimulation (ES) of phrenic nerves is a well-established technique [1-3]. However, this measurement is not widely used because ES can be technically difficult [4-6]. To overcome the problems of ES, cervical magnetic stimulation (CMS) $[5,6]$ and unilateral magnetic stimulation (UMS) of the phrenic nerve [4] have been developed. These techniques are both painless and easy to apply. However, the latencies [6, 7] and amplitudes [5] of the diaphragm compound muscle action potential (CMAP) measured with CMS are variable and different to those produced by ES. To evaluate the technique of UMS chest wall surface electrodes have previously been used to record the CMAP, and the PNCT was found to be shorter than that measured with ES [4]. The PNCT measured with an oesophageal electrode using UMS has not been reported, although it is considered that the diaphragm EMG recorded from an oesophageal electrode is more specific than when using surface electrodes [1]. The diaphragm EMG record- ed from chest wall electrodes can be contaminated by extradiaphragmatic muscle activity [2]; therefore, the PNCT measured with this method may be unreliable. To determine whether chest wall electrodes can be used to measure the diaphragm CMAP elicited by UMS and to establish normal values of PNCT, an oesophageal electrode and chest wall surface electrodes were used to record diaphragm CMAP. This study also investigated whether the phrenic nerves can be maximally stimulated by UMS.

\section{Methods}

\section{Subjects}

Eight healthy volunteers (five males and three females) aged 28-38 yrs (mean age 32 yrs) participated in the study. The subjects were members of staff and all were free of neurological and respiratory disease. The study was approved by the local ethical committee and subjects gave their informed consent. 


\section{Unilateral magnetic stimulation of the phrenic nerves}

UMS was performed using a double circular 43-mm coil (P/N 9784-00) powered by a Magstim 200 stimulator (Magstim Co., Whitland, Dyfed, UK). The coil was placed anterolaterally over the phrenic nerve [4]. During the study subjects were seated upright in a chair and stimulation was performed at end-expiration with the abdomen unbound.

\section{Electrical stimulation of the phrenic nerves}

The phrenic nerves were stimulated at the posterior border of the sternomastoid muscle at the level of the cricoid cartilage with a bipolar surface stimulating electrode (Medelec, Old Woking, Surrey, UK). The cathode was placed at the lower level. Square-wave impulses of $0.1-\mathrm{ms}$ duration were delivered. Stimulation began at low voltage. Once an action potential was observed the stimulus voltage was increased progressively until there was no further increase in amplitude of the CMAP. To ensure that supramaximal stimulation of the phrenic nerves was achieved, the intensity of stimulation was then further increased by $30 \%$ for the remainder of the study. The subjects adopted a similar position for ES to that for UMS. The stimulation was also delivered at end-expiration.

\section{Recording the compound muscle action potential}

Oesophageal electrode. The oesophageal electrode catheter consisted of three coils which were $1 \mathrm{~cm}$ in width, separated by a distance of $3 \mathrm{~cm}$. The middle electrode acted as a common electrode between the upper and lower electrodes to form two pairs of electrodes. Because the left and right hemidiaphragm are not at the same level and the electrical activity centres of each are usually 1-2 cm apart [1], a distance of $3 \mathrm{~cm}$ between electrodes was selected to include the electrical activity centre of both hemidiaphragms. The oesophageal electrode was introduced through the nose and swallowed into the oesophagus. To position the electrode over the centre of the electrically active region of the diaphragm (EARdi) [8], the common electrode was connected to the positive input terminal of the amplifier and the upper and lower electrodes were connected to negative input terminals. Because the shape and amplitude of the CMAP are sensitive to the electrode position [8], the common electrode was positioned over EARdi by observing the polarity and amplitude of the CMAP recorded from two pairs of electrodes during electrical stimulation. The ideal position was characterized by the same negative polarity and similar amplitude recorded from two pairs of electrodes following supramaximal ES. When the optimal position had been obtained the electrode catheter was fixed at the nose. The lower electrode pair was used to record the diaphragm CMAP for the remainder of the study. The purpose of the upper pair was to help to control the electrode position.

Surface electrode. Two skin silver/silver chloride electrodes (Arbo Medical, Stratford, CT, USA) were placed over roughened skin in a conventional fashion over the sixth to eighth intercostal space in the anterior axillary line on the left and right sides. The distance between electrodes was $3-5 \mathrm{~cm}$.

\section{Data acquisition}

EMG signals recorded from both the oesophageal and chest wall surface electrodes were amplified and band-pass filtered between $10 \mathrm{~Hz}$ and $10 \mathrm{kHz}$ (Magstim Co.). The signals were then passed to a 12-bit analogue-to-digital converter (NB-MIO-16; National Instruments, Austin, TX, USA) and stored and displayed on a Macintosh Centris Computer running Labview ${ }^{\mathrm{TM}} 2.2$ software (National Instruments). The signals were available in real time to the investigators. The sampling rate was $10 \mathrm{kHz}$.

\section{Study protocol}

Diaphragm compound muscle action potential recorded from oesophageal electrode. Diaphragm CMAP was obtained during UMS and ES. Five twitches were performed with supramaximal ES, and UMS at 40, 50, 60, 70, 80,90 and $100 \%$ of stimulator output.

Diaphragm compound muscle action potential recorded from surface electrodes. UMS at 60,80 and 100\% of stimulator output and supramaximal ES were delivered to elicit the CMAP. Again, five twitches were performed for ES and for each output level of magnetic stimulation. Five subjects participated in this study.

\section{Additional studies}

Study 1. Three patients with unilateral diaphragm paralysis were studied. Supramaximal ES and UMS at maximal magnetic stimulator output were performed and three twitches were delivered. The diaphragm CMAP was recorded from the oesophageal electrode. The purpose of this study was to determine whether magnetic stimulation of one phrenic nerve can depolarize the contralateral nerve.

Study 2. The diaphragm CMAP from the chest wall surface electrodes was also recorded in two patients with diaphragm paralysis during ES at an intensity of $150 \mathrm{~V}$ and UMS at maximal magnetic stimulator output. Five stimulations were performed for each technique. The purpose of this study was to observe whether chest wall surface electrodes can pick up CMAP from extradiaphragmatic muscles during UMS in patients with diaphragm paralysis.

Study 3. Contralateral and ipsilateral chest wall and central abdominal EMG were recorded simultaneously when stimulating one phrenic nerve with ES in three subjects. The abdominal electrodes were positioned 1 $\mathrm{cm}$ to the left and right of the navel. Five twitches were performed. The purpose of this study was to observe whether the CMAP can be detected at sites far away from the source of the electrical activity. 


\section{Data analysis}

The PNCT was defined as the time from stimulation artefact to the onset of the CMAP. The amplitude of the CMAP was measured from baseline to trough. t-Tests were used to test for differences throughout the study. Results were expressed as mean \pm SD.

\section{Results}

Both phrenic nerves were successfully assessed in the eight subjects. Six out of the eight subjects preferred UMS to ES. Two subjects complained of the noise generated by UMS when the magnetic stimulator output was over $90 \%$. The oesophageal electrode was well tolerated by all subjects. Magnetic stimulation of the phrenic nerves was easy to perform. With the coil placed laterally over the neck, over the phrenic nerve, optimal stimulation of the phrenic nerve was consistently achieved and it was not necessary to move the coil during studies.

Diaphragm electromyogram recorded from the oesophageal electrode

The CMAP elicited by ES was similar to that with UMS in polarity and shape (fig. 1). The baseline between the

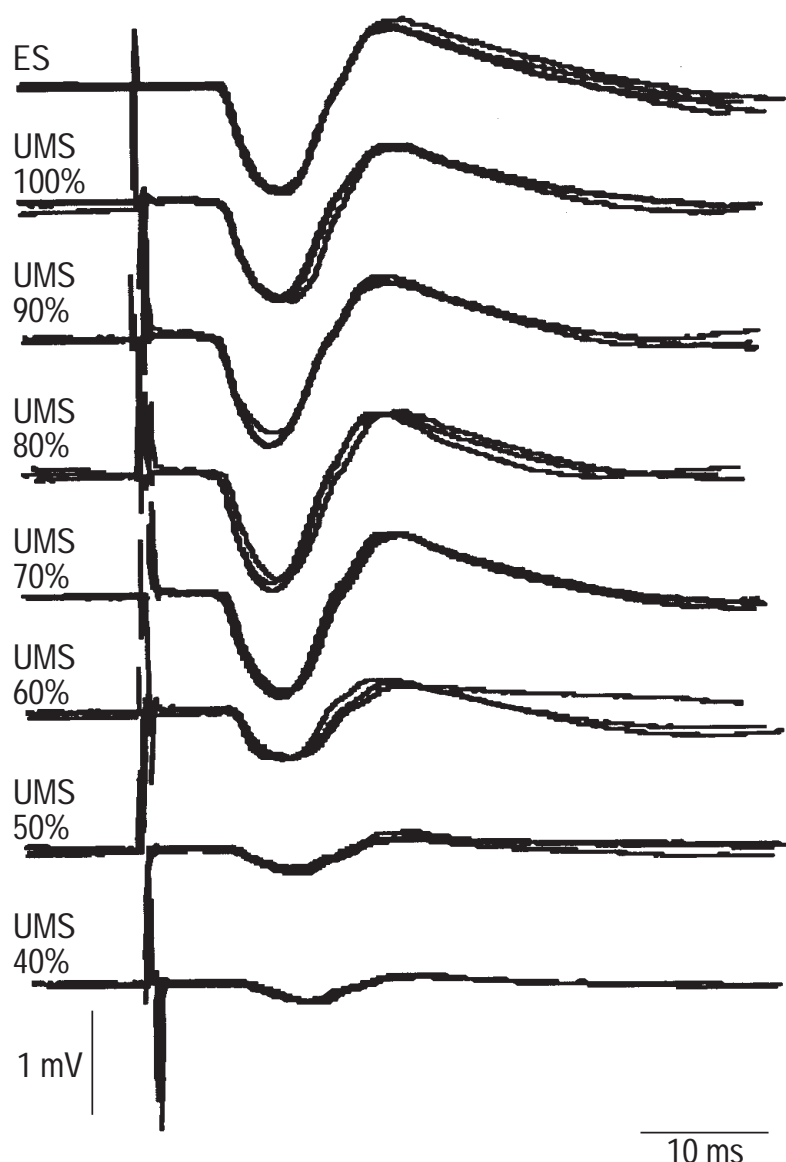

Fig. 1. - Compound muscle action potential (CMAP) recorded from the oesophageal electrode elicited by electrical stimulation (ES), and by unilateral magnetic stimulation (UMS) at different outputs. The amplitude of the CMAP increases with increasing stimulator output. The amplitude of the CMAP elicited with $80 \%$ output is similar to that elicited by ES. The shape of CMAP elicited by UMS at different stimulation outputs is similar to the shape of the CMAP elicited by ES. Three to five signals from one subject are superimposed.

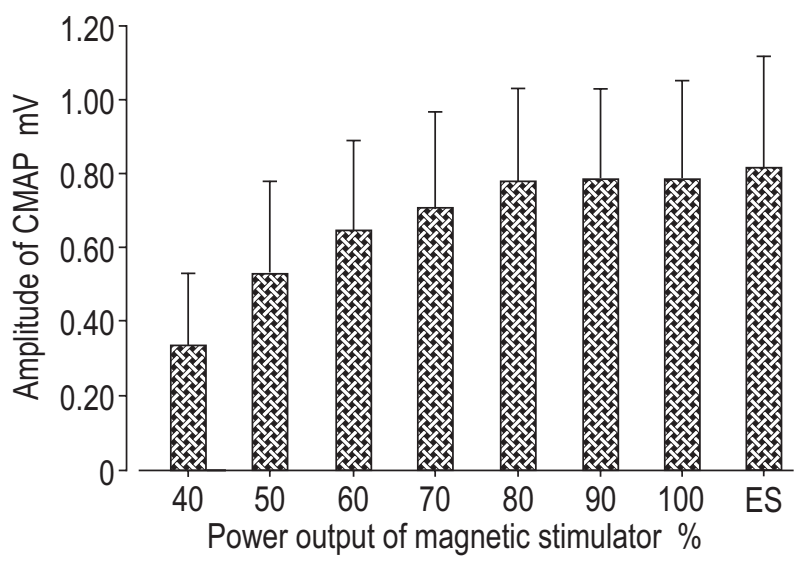

Fig. 2. - The diaphragm compound muscle action potential (CMAP) amplitude increases with increasing magnetic stimulator output. A clear plateau is achieved. The CMAP amplitude at the plateau is similar to the CMAP amplitude elicited by supramaximal electrical stimulation (ES). Data are pooled right and left values (mean $\pm \mathrm{SD}$ ).

stimulation artefact and the prominent downturn wave was as stable with UMS as with ES in most CMAP. The amplitude of the CMAP increased with increasing magnetic stimulator output for both the left and right sides. A plateau of the amplitude of the CMAP was achieved with increasing magnetic stimulator output (fig. 2). All phrenic nerves could be maximally stimulated. The mean amplitudes of the CMAP were, on the right side, $0.74 \pm 0.29 \mathrm{mV}$ for ES and $0.76 \pm 0.30 \mathrm{mV}$ for UMS with maximal power output, and on the left side $0.88 \pm$ $0.33 \mathrm{mV}$ for $\mathrm{ES}$ and $0.80 \pm 0.24 \mathrm{mV}$ for UMS ( $\mathrm{p}=\mathrm{NS}$ ). There were no significant differences in CMAP amplitude between the left and right sides for either ES or UMS.

PNCT measured with supramaximal ES was $7.8 \pm 1.2 \mathrm{~ms}$ for the left side and $7.0 \pm 0.8 \mathrm{~ms}$ for the right side. PNCT with UMS was similar to that measured with ES when the magnetic stimulator output was over $50 \%$; the PNCT measured with $40 \%$ output was slightly longer for the right side $(\mathrm{p}=0.04)($ table 1$)$.

Diaphragm electromyogram recorded from the chest wall surface electrodes

With ES the diaphragm EMG signals were of good quality for all subjects. The latencies measured with ES were $7.0 \pm 0.4 \mathrm{~ms}$ for the left side and $7.3 \pm 0.6 \mathrm{~ms}$ for right side. The latency of the CMAP measured with UMS was shorter, and shortened further with increasing stimulator output (fig. 3). The latency measured with UMS at 60, 80 and $100 \%$ magnetic stimulator output was $6.4 \pm 0.8$, $6.3 \pm 0.8$ and $4.6 \pm 0.6 \mathrm{~ms}$ for the left side and $6.0 \pm 1.1$, $5.1 \pm 0.7$ and $5.0 \pm 1.1 \mathrm{~ms}$ for the right, respectively. The latency measured with UMS at $100 \%$ stimulator output was significantly shorter than that measured with ES for both sides $(\mathrm{p}<0.05)$.

\section{Reproducibility}

Repeated measurements of latency with different intensities of magnetic stimulation were made in two subjects 1 month after the first study using oesophageal 
Table 1. - Phrenic nerve conduction time measured with different intensities of unilateral magnetic stimulation (UMS) and supramaximal electrical stimulation (ES)

\begin{tabular}{|c|c|c|c|c|c|c|c|c|}
\hline \multirow{2}{*}{$\begin{array}{l}\text { Subject } \\
\text { No. }\end{array}$} & \multicolumn{7}{|c|}{ UMS } & \multirow{2}{*}{ - ES } \\
\hline & $40 \%$ & $50 \%$ & $60 \%$ & $70 \%$ & $80 \%$ & $90 \%$ & $100 \%$ & \\
\hline \multicolumn{9}{|c|}{ Right side } \\
\hline 1 & 6.5 & 6.2 & 6.4 & 6.8 & 6.5 & 6.6 & 6.6 & 6.7 \\
\hline 2 & 6.5 & 6.3 & 6.2 & 6.2 & 6.2 & 6.1 & 6.5 & 6.4 \\
\hline 3 & 8.0 & 8.0 & 7.9 & 8.1 & 8.1 & 7.9 & 7.6 & 8.3 \\
\hline 4 & 7.5 & 7.0 & 7.0 & 7.3 & 7.4 & 7.1 & 7.1 & 6.9 \\
\hline 5 & 6.2 & 6.1 & 6.1 & 5.8 & 5.8 & 5.7 & 5.7 & 6.0 \\
\hline 6 & 7.9 & 7.7 & 7.6 & 7.5 & 7.8 & 7.6 & 7.8 & 7.5 \\
\hline 7 & 9.0 & 9.0 & 7.4 & 7.5 & 7.3 & 7.4 & 8.0 & 7.8 \\
\hline 8 & 6.8 & 6.5 & 6.4 & 6.1 & 6.0 & 6.1 & 6.1 & 6.3 \\
\hline Mear & 7.3 & 7.1 & 6.9 & 6.9 & 6.9 & 6.8 & 6.9 & 7.0 \\
\hline SD & 1.0 & 1.0 & 0.7 & 0.8 & 0.9 & 0.8 & 0.8 & 0.8 \\
\hline
\end{tabular}

\begin{tabular}{lcccccccc}
\hline Left side & & & & & & & & \\
1 & 6.6 & 6.4 & 6.4 & 6.4 & 6.4 & 6.4 & 6.3 & 6.8 \\
2 & 6.7 & 6.8 & 6.9 & 6.9 & 6.9 & 7.0 & 6.6 & 6.6 \\
3 & 8.8 & 8.7 & 8.9 & 9.0 & 8.8 & 8.9 & 8.7 & 8.4 \\
4 & 6.8 & 7.1 & 6.9 & 6.7 & 6.4 & 6.4 & 6.2 & 6.4 \\
5 & 7.3 & 7.4 & 7.3 & 7.4 & 7.2 & 7.4 & 7.5 & 7.3 \\
6 & 10.0 & 9.8 & 9.8 & 9.9 & 9.7 & 9.9 & 9.7 & 9.7 \\
7 & 10.0 & 9.3 & 9.1 & 9.3 & 9.2 & 9.3 & 9.1 & 9.2 \\
8 & 8.2 & 8.1 & 7.8 & 7.9 & 7.7 & 7.6 & 7.7 & 7.7 \\
\hline Mean & 8.0 & 8.0 & 7.9 & 7.9 & 7.8 & 7.9 & 7.7 & 7.8 \\
SD & 1.4 & 1.2 & 1.2 & 1.3 & 1.3 & 1.3 & 1.3 & 1.2 \\
\hline
\end{tabular}

electrodes. The latencies were no different between the two studies with UMS at $60 \%$ output $(6.5 \pm 0.3$ for the first study versus $6.6 \pm 0.2 \mathrm{~ms}$ for second, pooled right and left values), $80 \%$ output $(6.5 \pm 0.3$ versus $6.5 \pm 0.4 \mathrm{~ms}), 100 \%$ output $(6.5 \pm 0.2$ versus $6.6 \pm 0.2 \mathrm{~ms})$ and $\mathrm{ES}(6.6 \pm 0.2$ versus $6.6 \pm 0.2 \mathrm{~ms})$.

\section{Additional studies}

Study 1. In the three patients no CMAP could be recorded from the oesophageal electrode during stimulation of the affected side with ES. This supported the diagnosis of hemidiaphragm paralysis. With UMS no CMAP could be recorded from the oesophageal electrode when stimulating the affected side, whereas a clear CMAP was recorded when stimulating the normal side (fig. 4).

Study 2. A small CMAP with a short latency was recorded from chest wall surface electrodes in patients with diaphragm paralysis during UMS but no CMAP were recorded during ES (fig. 5).

Study 3. In two of the three normal subjects, a small potential ( $8 \%$ of ipsilateral CMAP amplitude) could be recorded from the contralateral chest wall electrodes during ES. A bigger CMAP (18\% of ipsilateral CMAP amplitude) was recorded from the central abdominal electrodes during unilateral ES (fig. 6) in all three subjects.

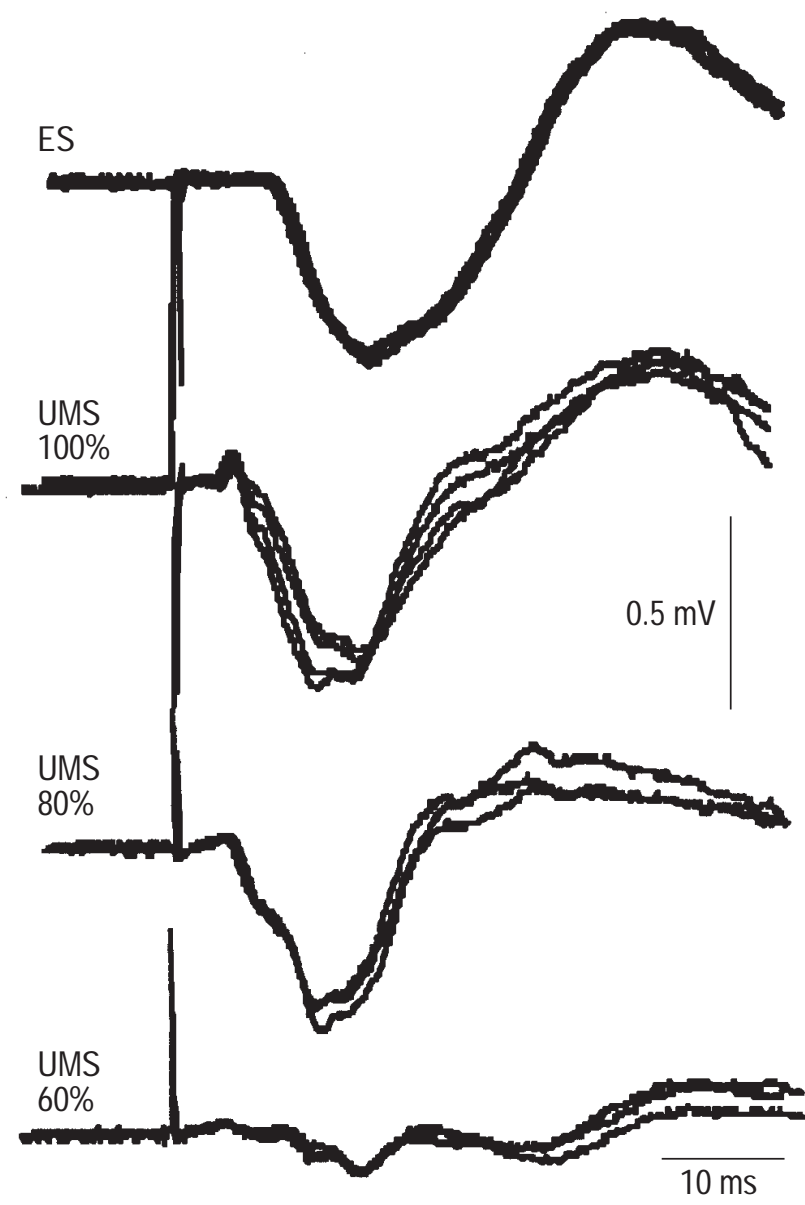

Fig. 3. - Compound muscle action potential (CMAP) recorded from chest wall surface electrodes, elicited by electrical stimulation (ES) or unilateral magnetic stimulation (UMS) at different outputs. There is a small wave before the prominant negative wave of the CMAP elicited by UMS at $100 \%$ output. The trace of the UMS-CMAP is irregular. The latency of the CMAP measured with UMS is much shorter than that elicited by ES. Three to five signals from one subject are superimposed.

\section{Discussion}

In this study the PNCT measured with UMS was comparable to that measured with ES when the diaphragm CMAP was recorded from an oesophageal electrode. In addition, a clear plateau of the amplitude of the CMAP was achieved with increasing magnetic stimulation intensity. The latency of the CMAP measured with UMS was shorter than that measured with ES for chest wall recordings.

The amplitude of the CMAP increased with increasing magnetic stimulator output and this result was similar to a previous study [4]. However, the stimulus intensity required to achieve maximal phrenic nerve stimulation in the present study was lower. This cannot be explained by the magnetic stimulator itself because the stimulator used was the same for both investigations. The likely explanation is the use of oesophageal rather than surface electrodes.

It is interesting to compare CMS with UMS in the assessment of diaphragm function. Firstly, with CMS the amplitude [5] and shape [9] of the CMAP are sometimes different to those elicited with ES. It is also often difficult to demonstrate a plateau with increasing stimulus intensity with CMS [10]. Even with maximal stimulator output 

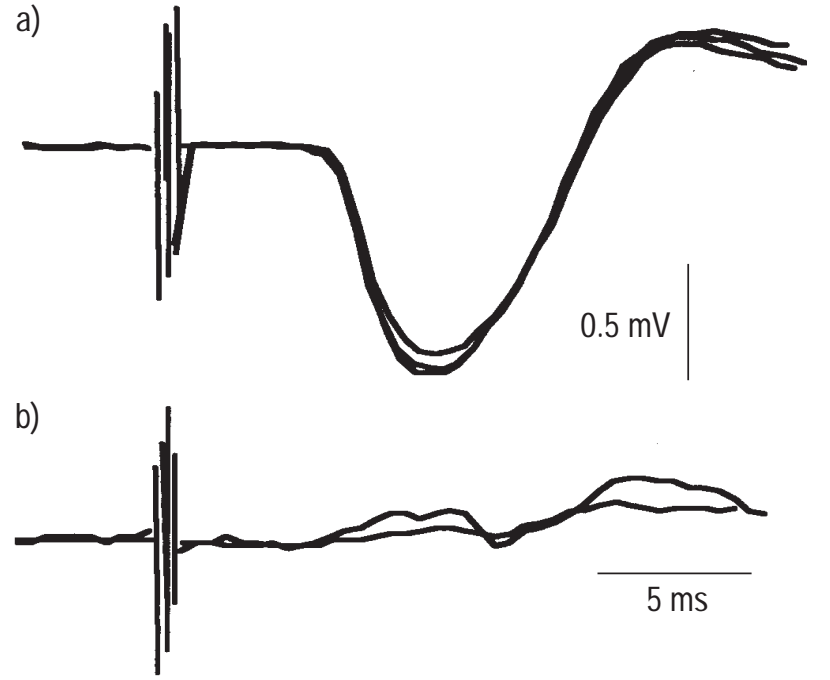

Fig. 4. - Diaphragm compound muscle action potential (CMAP) elicited by unilateral magnetic stimulation (UMS) at $100 \%$ output recorded from a patient with unilateral diaphragm paralysis. a) Normal side; and b) paralysed side. Two to three signals are superimposed.

CMS cannot always achieve a maximal twitch response either in terms of diaphragmatic pressure $(P \mathrm{di})$ or diaphragm CMAP [11], reducing the utility of the technique for assessing diaphragm function [12]. By contrast, the shape and amplitude of the CMAP elicited by UMS is similar to that elicited by ES and a clear plateau of the amplitude of the CMAP can be achieved with increasing magnetic stimulation intensity. These results suggest that UMS can maximally stimulate the phrenic nerve and is genuinely as effective as ES in eliciting the diaphragm twitch response. Secondly, UMS can selectively stimulate one phrenic nerve and hemidiaphragm function can be assessed. This is not possible with CMS. Thirdly, UMS can be used to measure unilateral PNCT, whereas it is not possible with CMS to distinguish left from right PNCT when using an oesophageal electrode and the use of chest wall electrodes with CMS is difficult [10]. UMS is an easy method of phrenic nerve stimulation. The area for stimulation is sufficiently large $(4 \times 4.5 \mathrm{~cm})$ that excitation of the phrenic nerve can be achieved reliably and constantly.

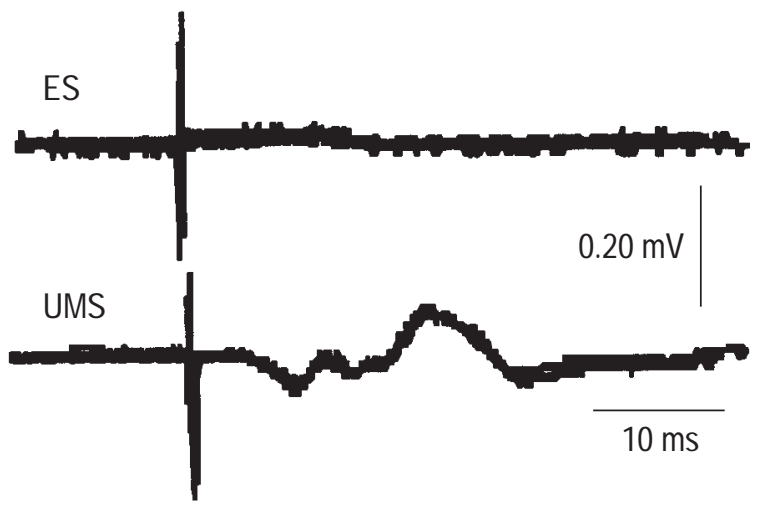

Fig. 5. - Compound muscle action potential (CMAP) recorded from surface electrodes during electrical stimulation (ES) and unilateral magnetic stimulation (UMS) in a patient with diaphragm paralysis. No potential is recorded during ES, while a small potential with a short latency $(4.5 \mathrm{~ms})$ is recorded during UMS. Three signals are superimposed.
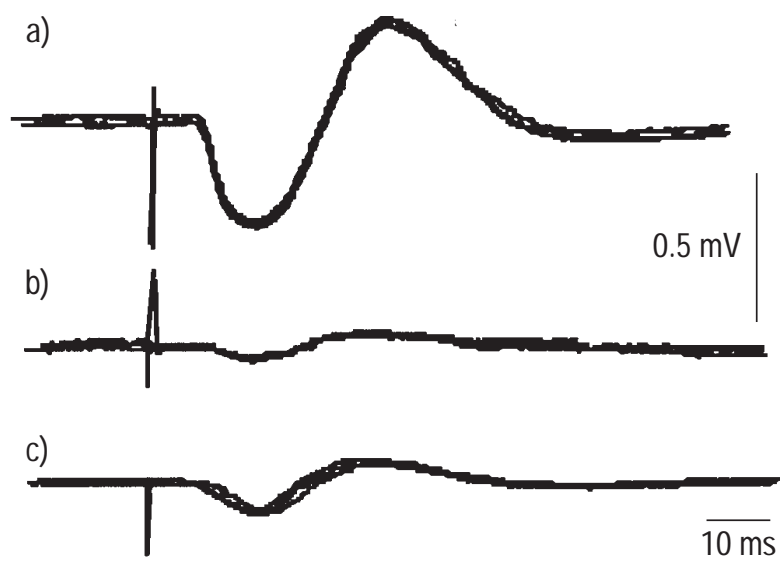

Fig. 6. - Diaphragm compound muscle action potential (CMAP) recorded simultaneously from ipsilateral and contralateral chest wall electrodes and centre of the abdomen. a) Ipsilateral diaphragm CMAP; b) contralateral diaphragm CMAP; and c) diaphragm CMAP recorded from the abdomen. Three to five signals from one subject are superimposed.

The original study of unilateral magnetic stimulation suggested that it can stimulate the contralateral phrenic nerve, as assessed from the diaphragm EMG recorded from surface electrodes [4]. If this were correct the usefulness of UMS in the diagnosis of neuromuscular disease, such as unilateral diaphragm paralysis, would be reduced. However, if UMS does stimulate the contralateral phrenic nerve, the amplitude of the CMAP elicited by increasing intensity of UMS will gradually increase as more and more phrenic nerve fibres on the contralateral side are depolarized. In the present study, with the specially designed oesophageal electrode, the clear plateau of the amplitude of the CMAP with increasing stimulator output suggests that contralateral phrenic nerve stimulation does not occur. More importantly, no diaphragm CMAP could be recorded when stimulating the nonfunctioning phrenic nerve with UMS at maximal stimulator output.

It has been suggested that magnetic stimulation preferentially depolarizes fast fibres in peripheral nerves and this could explain the difference between ES-CMAP and UMS-CMAP. However, any preferential recruitment of fast fibres would only be relevant for submaximal stimulation. For supramaximal stimulation of the phrenic nerve all fibres will be depolarized. If the depolarized point of the phrenic nerve is the same for both ES and magnetic stimulation, the latency of the CMAP would be expected to be the same. Indeed, the CMAP elicited by ES and magnetic stimulation of a peripheral nerve is similar. MACCABEE et al. [13] investigated median nerve electrical and magnetic stimulation and found that the latency measured with electrical stimulation was similar to that measured with magnetic stimulation when the stimulation point was the same. The shape of the CMAP elicited by magnetic stimulation was similar to that elicited by electrical stimulation when maximal stimulation was achieved. In the present study the latency and shape of the CMAP measured with ES were also similar to those measured with UMS when using the oesophageal electrode, suggesting that UMS is similar to ES when both are supramaximal.

Chest wall electrodes have been widely used to record the diaphragm CMAP with ES because of convenience and 
acceptability by subjects. However, whether surface electrodes can always record a pure diaphragm CMAP elicited by magnetic stimulation is controversial. SIMILOWSKI et al. [7] measured PNCT with surface electrodes using CMS and reported much shorter values than with ES. LAGHI et al. [5] reported that the diaphragm CMAP elicited by CMS was sometimes much higher than that elicited by supramaximal ES. MiLls et al. $[4,11]$ noted that the chest wall surface diaphragm EMG elicited by magnetic stimulation of the phrenic nerves was sometimes different from that elicited by ES and the latency measured with UMS was shorter than that measured with ES, which they considered to be due to the diaphragm EMG being contaminated by extradiaphragmatic muscle activity. In the present study, the latency of the CMAP measured with UMS was also shorter than that with ES using chest wall surface electrodes. In contrast, the results were similar using oesophageal electrodes. Furthermore, no obvious action potential could be recorded from chest wall electrodes during focused ES in patients with diaphragm paralysis, whereas a small, short-latency CMAP was recorded during UMS (fig. 5). These results suggest that the diaphragm CMAP elicited by UMS recorded from surface electrodes may be interfered with by short-latency potentials. Because the latency of CMAP of chest wall muscles is usually shorter than that of the diaphragm [2], it can be hypothesized that the chest wall diaphragm CMAP elicited by UMS is contaminated by the activity of chest wall muscles.

The CMAP can be transmitted to distant electrodes by volume conduction. It has been shown that a CMAP can be recorded from electrodes placed on the hypothenar eminence, far from any actively contracting muscle during stimulation of the median nerve at the wrist [2]. BELLEMARE and BigLAND-Ritchie [14] demonstrated that the diaphragm CMAP can be recorded from contralateral chest wall electrodes when stimulating one phrenic nerve with ES, an observation which is confirmed by the present study. Furthermore, the diaphragm CMAP could be recorded at the centre of the abdomen, far from the diaphragm, after ES (fig. 6). In principle, the activity of chest wall muscles (pectoralis major and serratus anterior) adjacent to the electrodes could also contribute to the surface diaphragm EMG by volume conduction. Therefore, the short-latency CMAP elicited by magnetic stimulation recorded from surface electrodes may be due to the diaphragm CMAP being contaminated by extradiaphragmatic muscle activity. Indeed, even with the relatively focused technique of ES, the diaphragm CMAP recorded from chest wall electrodes may not always be free of contamination from extradiaphragmatic muscle activity. McKenzIE and GANDEVIA [1] found that in two out of 20 subjects they could not reliably measure the CMAP because of probable contamination from other muscles. MARKAND et al. [2] emphasized that the diaphragm EMG elicited by ES recorded from electrodes placed in the anterior or posterior axillary line in the eighth intercostal space was probably contaminated by motor responses of other chest muscles. These studies suggest that PNCT measured with chest wall surface electrodes is likely to be unreliable when using magnetic stimulation, which unavoidably activates many chest muscles during phrenic nerve stimulation. Although SIMILOWSKI et al. [7] reported that they could not record any CMAP from a patient with bilateral diaphragm paralysis, the patients they studied had severe amyotrophic lateral sclerosis (ALS) and, therefore, the absent CMAP with CMS could have been due to the denervation of the chest wall muscles.

In conclusion, unilateral magnetic stimulation is as effective as electrical stimulation in maximally stimulating the phrenic nerve. The phrenic nerve conduction time can be measured with unilateral magnetic stimulation, providing an oesophageal electrode is used. The diaphragm compound muscle action potential elicited by unilateral magnetic stimulation recorded from chest wall surface electrodes is unreliable.

\section{References}

1. McKenzie DK, Gandevia SC. Phrenic nerve conduction time and twitch pressures of the human diaphragm. $J$ Appl Physiol 1985; 58: 1496-1504.

2. Markand ON, Kincaid JC, Pourmand RA, et al. Electrophysiologic evaluation of diaphragm by transcutaneous phrenic nerve stimulation. Neurology 1984; 34: 604-614.

3. Chen R, Collins S, Remtulla H, Parkes A, Bolton CF. Phrenic nerve conduction study in normal subjects. Muscle Nerve 1995; 18: 330-335.

4. Mills GH, Kyroussis D, Hamnegard CH, Wragg S, Moxham J, Green M. Unilateral magnetic stimulation of the phrenic nerve. Thorax 1995; 50: 1162-1172.

5. Laghi F, Harrison MJ, Tobin MJ. Comparison of magnetic and electrical phrenic nerve stimulation in assessment of diaphragm contractility. J Appl Physiol 1996; 80: 17311742.

6. Similowski T, Fleury B, Launois S, Cathala HP, Bouche P, Derenne JP. Cervical magnetic stimulation: a new painless method for bilateral phrenic nerve stimulation in conscious humans. J Appl Physiol 1989; 67: 1311-1318.

7. Similowski T, Mehiri S, Duguet A, Attali V, Straus C, Derenne JP. Comparison of magnetic and electrical phrenic nerve stimulation in assessment of phrenic nerve conduction time. J Appl Physiol 1997; 82: 1190-1199.

8. Beck J, Sinderby C, Lindstrom L, Grassino A. Diaphragm interference EMG and compound muscle action potentials: effects of chest wall configuration. J Appl Physiol 1997; 80: 520-530.

9. Similowski T, Duguet A, Straus C, Attali V, Boisteanu D, Derenne JP. Assessment of the voluntary activation of the diaphragm using cervical and cortical magnetic stimulation. Eur Respir J 1996; 9: 1224-1231.

10. Mador MJ, Rodis A, Magalang UJ, Ameen K. Comparison of cervical magnetic and transcutaneous phrenic nerve stimulation before and after threshold loading. Am J Respir Crit Care Med 1996; 154: 448-453.

11. Mills GH, Kyroussis D, Hamnegard CH, Polkey MI, Green M, Moxham J. Bilateral magnetic stimulation of phrenic nerves from an anterolateral approach. Am J Respir Crit Care Med 1996; 154: 1099-1105.

12. McKenzie DK, Gandevia SC. Electrical assessment of respiratory muscles. In: Roussos C, ed. The Thorax. 2nd Edn. New York, Marcel Dekker, 1995; pp. 1029-1048.

13. Maccabee PJ, Amassian VE, Cracco RQ, Cadwell JA. An analysis of peripheral motor nerve stimulation in humans using the magnetic coil. Electroencephalogr Clin Neurophysiol 1988; 70: 524-533.

14. Bellemare F, Bigland-Ritchie B. Assessment of human diaphragm strength and activation using phrenic nerve stimulation. Respir Physiol 1984; 58: 263-277. 